\title{
Validation of Internal Charging Tools With Experiments in REEF
}

\author{
Alex D. P. Hands ${ }^{\circledR}$, Member, IEEE, Keith A. Ryden ${ }^{\circledR}$, Member, IEEE, Remi Pacaud ${ }^{\circledR}$, \\ Thierry Paulmier ${ }^{(\mathbb{1}}$, Pierre Sarrailh ${ }^{(\mathbb{D}}$, Denis Payan ${ }^{(}$, and David Rodgers
}

\begin{abstract}
The objective of the European Space Agency validation of internal charging tools using the realistic electron environmental facility (REEF) project is to assess the performance of internal charging tools against experimental measurements made at the REEF facility at the University of Surrey. REEF uses an intense strontium-90 beta-emitting radioactive source to simulate the space environment. This project is complemented by parallel experiments to derive material parameters, conducted by ONERA. We report results from REEF with four different types of dielectric material and compare these results to predictions from the DICTAT, MCICT, and NUMIT internal charging simulation tools. The materials under investigation are Cirlex, PEEK, FR4, and Neoflon (FEP). We find that in many cases, the computer codes struggle to recreate REEF results, which raises significant questions over the validity of internal charging mitigation analyses. We show the advantages and disadvantages of each model and suggest what features could be added in order to improve the fidelity of their predictions.
\end{abstract}

Index Terms-Electrostatic discharge (ESD), ground radiation testing, internal charging.

\section{INTRODUCTION}

$\mathbf{I}$ NTERNAL charging of dielectric materials within spacecraft remains a major cause for concern. The risk posed to the survivability of satellite systems by the populations of trapped energetic electrons in the Van Allen belts has long been established [1]. High-energy electrons are able to penetrate the outer layers of a spacecraft and become trapped in insulating materials, sometimes leading to unsustainably high electric fields and electrostatic discharges (ESDs). Although the ESD phenomenon itself is well understood [2], the charging response of materials in a rapidly varying electron environment is less well characterized. The ESD threat is mitigated by the radiation-induced conductivity (RIC) from ionizing dose

Manuscript received September 11, 2018; revised January 11, 2019; accepted February 18, 2019. This work was supported by the European Space Agency under ESA Contract 4000121891/17/NL/LF/as. The review of this paper was arranged by Senior Editor H. B. Garrett. (Corresponding author: Alex D. P. Hands.)

A. D. P. Hands and K. A. Ryden are with the Surrey Space Centre, University of Surrey, Guildford GU2 7XH, U.K. (e-mail: a.hands@surrey.ac.uk)

R. Pacaud, T. Paulmier, and P. Sarrailh are with ONERA/DPHY, Université de Toulouse, 31055 Toulouse, France.

D. Payan is with the Centre National d'Études Spatiales, 31400 Toulouse, France.

D. Rodgers is with the European Space Research and Technology Centre, European Space Agency, 2200 AG Noordwijk, The Netherlands.

Color versions of one or more of the figures in this paper are available online at http://ieeexplore.ieee.org.

Digital Object Identifier 10.1109/TPS.2019.2900891 caused by the same electron environment. Thus, the threat due to internal charging depends very much on the rate at which electrons are deposited in a dielectric material (the charging current) as well as the total cumulative charge deposition. This makes accelerated testing with laboratory facilities problematic, as a benign response to such an intense environment may lead to a false sense of security over the equivalent build-up of electric fields in a real environment. Exhaustive testing of materials in a more realistic environment is one way to circumvent this problem, but this necessarily involves very long duration experiments and, thus potentially, very high costs. Such lengthy experiments also reduce the scope for the number of permutations of test conditions that could bound the risk of charging. Therefore, the risk assessment process for internal charging often depends on the results of computer simulations that aim to predict the response of dielectric materials given an assortment of input parameters. These input parameters include intrinsic properties of the dielectrics themselves, as well as the characterization of the electron environment and the geometrical configuration of the sensitive material. The fidelity of the simulation code output depends both on the validity of the input parameters and the underlying physics in the model [3].

\section{EXPERIMENTS}

The realistic electron environmental facility (REEF), located at the University of Surrey, exposes samples in vacuum to a $\sim 2.5-\mathrm{GBq}$ Sr-90 source (activity calculated for October 2017). Strontium-90 provides an excellent practical option for the provision of long-duration, low-intensity exposures as it allows uninterrupted irradiations over the required long periods with an electron spectrum that is approximately representative of the real space environment. Fig. 1 shows the REEF facility setup at the university (prior to the addition of a protective cage that was added for security and safety considerations). The intensity of the electron environment within the facility is controlled via varying the source-sample separation distance and, if necessary, adding shielding to attenuate the electron spectrum. The dynamic range of incident electron current achievable with REEF is wide, ranging from $\sim 6 \mathrm{pA} / \mathrm{cm}^{2}$ at low $(\sim 3.5 \mathrm{~cm})$ source-sample separation to $\sim 0.3 \mathrm{pA} / \mathrm{cm}^{2}$ at the maximum source-sample separation $(\sim 16 \mathrm{~cm})$. Further reductions in current can be achieved by adding planar aluminum shielding in between source and sample. A Trek probe is maneuvered horizontally to a position directly in between 


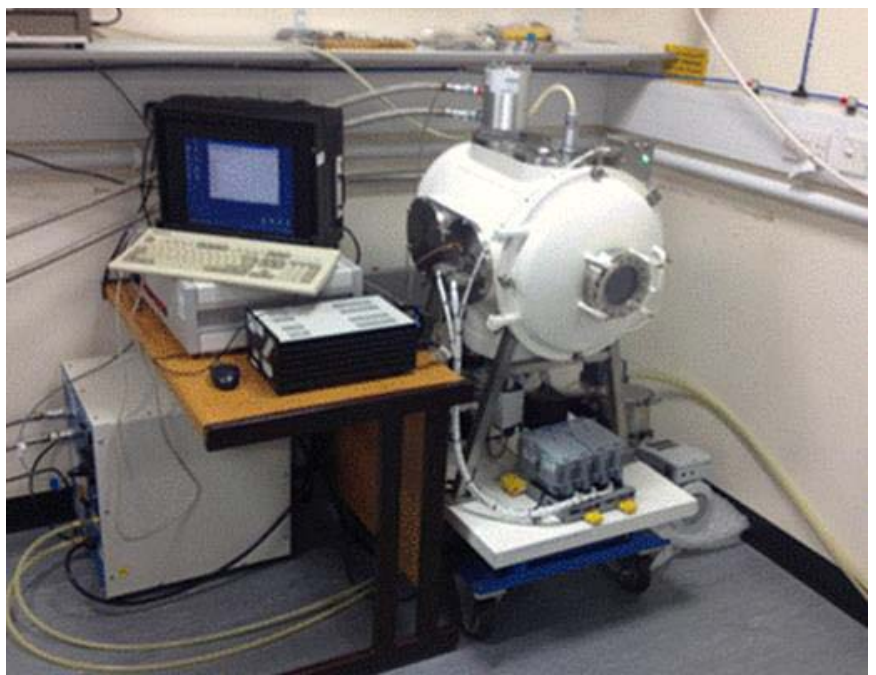

Fig. 1. REEF equipment assembled at the University of Surrey. The equipment is shown before the installation of a protective cage.

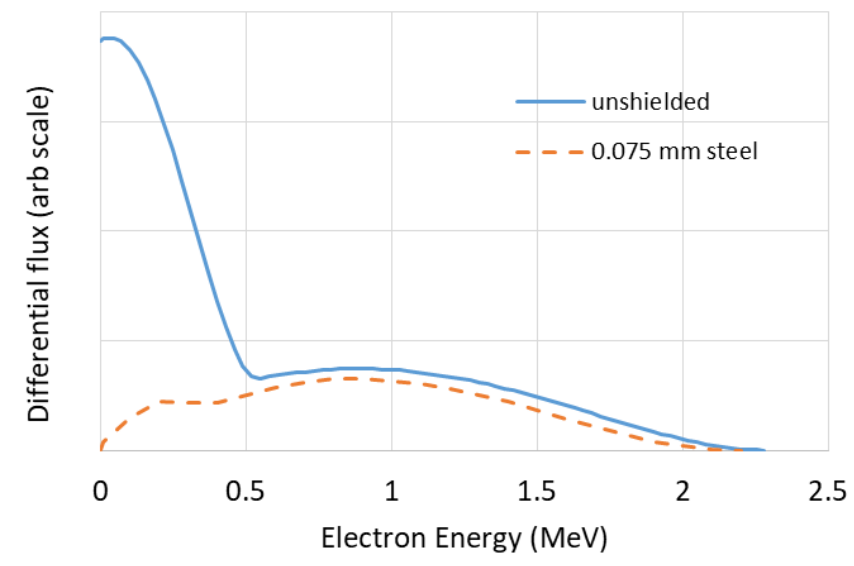

Fig. 2. REEF electron spectrum before and after attenuation by a thin stainless steel window on the source packaging. The "after" spectrum was calculated using MULASSIS simulations.

the source and sample and automatically takes measurements of surface potential from the irradiated sample every hour (up to a maximum negative value of $\sim 3400 \mathrm{~V}$ ). More detail can be found in [4] and [5].

REEF is capable of continuous long-duration observations of the charging response of dielectric materials under irradiation [5]. The incident spectrum on samples placed inside REEF is modified by a thin $(0.075 \mathrm{~mm})$ stainless steel window that forms part of the packaging of the sealed Sr-90 source. This has a significant impact on the lower energy part of the electron spectrum, reducing the total incident flux over all energies by approximately $60 \%$. We have used the GEANT4 tool MULASSIS [6] to simulate the effect of the stainless steel layer on the theoretical $\mathrm{Sr}-90$ spectrum [7]. The results are shown in Fig. 2.

\section{A. Experimental Setup}

The charging response of dielectric materials to incident electrons is dependent on a number of factors, including physical and dielectric properties of the material, ambient temperature, grounding configuration, pressure, and intensity.
TABLE I

Default Physical and Dielectric Parameters For the Four MATERIALS UsEd IN THE VICTOR TEST CAMPAIGN

\begin{tabular}{|c|c|c|c|c|}
\hline Property & Cirlex & Neoflon & FR4 & PEEK \\
\hline $\begin{array}{l}\text { Thickness } \\
\text { (mm) }\end{array}$ & 1.0 & 0.5 & 1.5 & 0.5 \\
\hline $\begin{array}{l}\text { Molecular } \\
\text { formula }\end{array}$ & $\mathrm{C}_{22} \mathrm{H}_{10} \mathrm{~N}_{2} \mathrm{O}_{5}$ & $\mathrm{C}_{2} \mathrm{~F}_{3} \mathrm{Cl}$ & $\mathrm{Si}_{10} \mathrm{O}_{25} \mathrm{C}_{21} \mathrm{H}_{25} \mathrm{Cl}$ & $\mathrm{C}_{21} \mathrm{H}_{18} \mathrm{O}_{3}$ \\
\hline$Z$ effective* & 6.36 & 10.79 & 9.33 & 6.02 \\
\hline A effective* & 12.40 & 21.77 & 18.21 & 11.39 \\
\hline $\begin{array}{l}\text { Density } \\
(\mathrm{g} / \mathrm{cm} 3)\end{array}$ & 1.42 & 2.15 & 1.85 & 1.3 \\
\hline $\begin{array}{l}\text { Relative } \\
\text { permittivity } \\
\left(\varepsilon_{r}\right)\end{array}$ & 3.45 & 2.1 & 4.7 & 3.3 \\
\hline $\begin{array}{l}\text { Bulk } \\
\text { Conductivity } \\
\text { (S/m) }\end{array}$ & $<10^{-15}$ & $<10^{-15}$ & $3 \times 10^{-15}$ & $<10^{-15}$ \\
\hline $\begin{array}{l}\mathbf{k}_{\mathrm{p}}(\mathrm{S} / \mathrm{m} \\
\left.[\mathrm{rad} / \mathrm{s}]^{-1}\right)\end{array}$ & $4.40 \mathrm{E}-15$ & $8.73 \mathrm{E}-14$ & 0 & $5.00 \mathrm{E}-15$ \\
\hline$\Delta$ & 1 & 0.47 & - & 1 \\
\hline
\end{tabular}

As small temperature fluctuations can have a significant effect on the charging profiles of dielectrics, we decided to maintain a constant temperature for our experiments. In order to circumvent the lack of temperature control in the REEF laboratory, we used the sample heating capability of REEF to conduct irradiations at a constant temperature of $310 \mathrm{~K}$ (as this is above the maximum diurnal ambient fluctuation). This applies to all results presented in this paper. The REEF chamber has two vacuum pumps that together are capable of achieving an operational pressure as low as $\sim 10^{-5}$ mbar. Reaching such a high vacuum can take as long as $24 \mathrm{~h}$, on the other hand, a pressure of $\sim 10^{-4}$ mbar can be achieved in less than $2 \mathrm{~h}$. In order to minimize the preparation time, and in accordance with the operational procedure in previous REEF experimental campaigns, we allow commencement of irradiation when the pressure of the chamber has been reduced to approximately $10^{-4}$ mbar.

\section{B. Materials Under Test}

ONERA procured planar samples of four dielectric materials, for irradiation at their SIRENE and GEODUR facilities [8]. The materials are Cirlex, Neoflon (FEP), FR4, and PEEK. These have been selected on the basis of availability, applicability to spacecraft design, and existing knowledge of dielectric parameters. From the irradiations, material parameters related to bulk and RIC (RIC parameters are $k_{\mathrm{p}}$ and $\Delta$ ) were derived. These are given in Table I alongside physical properties that are relevant to the simulations later in this paper.

A subset of these samples was provided to the University of Surrey for irradiation in REEF. All samples had been metalized on one side so that they could be grounded to the REEF sample holder. Each sample is $30 \mathrm{~mm} \times 50 \mathrm{~mm}$ in size.

\section{Incident Current}

The decision on which the initial current level to select for a given material was taken on a "best guess" of the response based on the experience of previous campaigns. Current levels for subsequent exposures were selected using the initial data 
TABLE II

SUMMARY OF EXPOSURES COMPLETED IN THE VICTOR EXPERIMENTAL CAMPAIGN

\begin{tabular}{|l|c|c|c|c|}
\hline Current & Cirlex & Neoflon & FR4 & PEEK \\
\hline $\begin{array}{l}\text { High } \\
\left(\mathbf{5 ~ p A} / \mathrm{cm}^{2}\right)\end{array}$ & & $\checkmark$ & $\checkmark$ & $\checkmark$ \\
\hline $\begin{array}{l}\text { Medium } \\
\left(\mathbf{1} \mathrm{pA} / \mathrm{cm}^{2}\right)\end{array}$ & $\checkmark$ & $\checkmark *$ & $\checkmark$ & $\checkmark *$ \\
\hline $\begin{array}{l}\text { Low } \\
\left(\mathbf{0 . 3} \mathbf{~ p A} / \mathrm{cm}^{2}\right)\end{array}$ & $\checkmark$ & $\checkmark * *$ & & $\checkmark *$ \\
\hline $\begin{array}{l}\text { Shielded } \\
\left(\mathbf{0 . 1 6} \mathbf{~ p A} / \mathrm{cm}^{2}\right)\end{array}$ & $\checkmark$ & & $\checkmark$ \\
\hline \\
*Extended with a high current exposure. \\
**Repeated to test for out-gassing effects.
\end{tabular}

as a guide. In two cases (once each for Cirlex and PEEK), the current was further reduced than the minimum of our normal dynamic range $\left(0.3 \mathrm{pA} / \mathrm{cm}^{2}\right)$ using a thin piece of aluminum shielding to attenuate the Sr-90 spectrum. Using the GEANT4 to propagate the current through this additional shielding, we calculate the current to be approximately $0.16 \mathrm{pA} / \mathrm{cm}^{2}$.

\section{RESULTS}

In total, 13 samples were exposed to the REEF environment as part of the validation of internal charging tools using REEF (VICToR) project. This total is comprised of $2 \times$ FR4, $3 \times$ Cirlex, $4 \times$ Neoflon, and $4 \times$ PEEK. A summary of the current levels for these experiments is given in Table II. One of the Neoflon exposures was a repeat experiment to test for out-gassing effects by allowing the sample to settle in vacuum for 2 days prior to exposure to the source. This revealed no significant difference so this settling period was deemed unnecessary for future exposures. In addition, two PEEK exposures and one Neoflon exposure were extended with higher current levels, for comparison with exposures of fresh samples at these levels.

Full surface voltage measurements are given below for each material. In many cases, when samples were initially placed inside the REEF chamber, they exhibited a surface voltage of several tens or even several hundreds of volts. This was presumed likely because of the triboelectric effect on surface electrons rather than internal trapped charge. In order to dissipate this charge, prior to pumping down the vacuum chamber, the source holder was opened for approximately $1 \mathrm{~min}$. This allows the ionization of the air and the removal of lightly bound surface charge. Due to their brevity and the fact that an aluminum shield was placed in between the source and sample, these preliminary exposures had no effect on the internal charging sample response of the samples. On the commencement of the full exposures under vacuum, the initial surface voltage was sometimes still up to $\sim 10 \mathrm{~V}$ from 0 . It is unclear whether this residual charge is an undissipated surface charge or another factor, but it is negligible compared to subsequent accumulated charge so no renormalization has been applied to the surface voltages plotted below.

\section{A. Cirlex Results}

Exposures of Cirlex, a dielectric with similar properties to Kapton, were conducted at low, medium, and shielded current

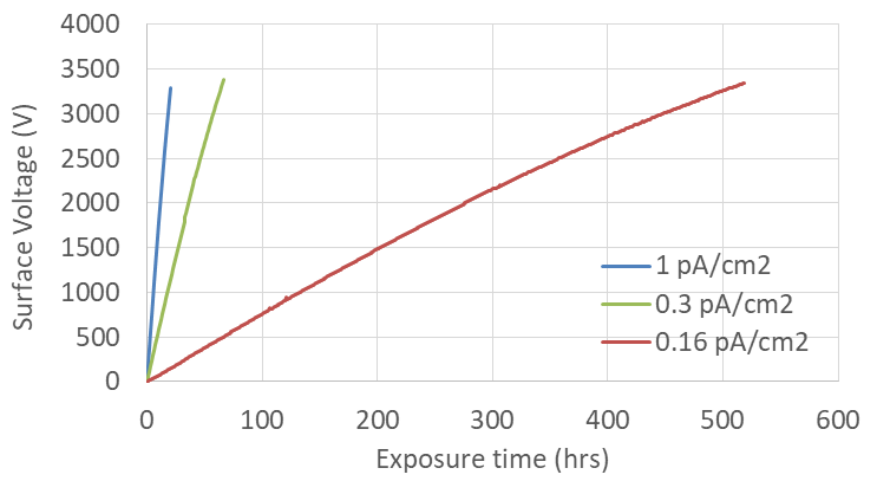

Fig. 3. Surface voltage measurements as a function of time for Cirlex samples in REEF.

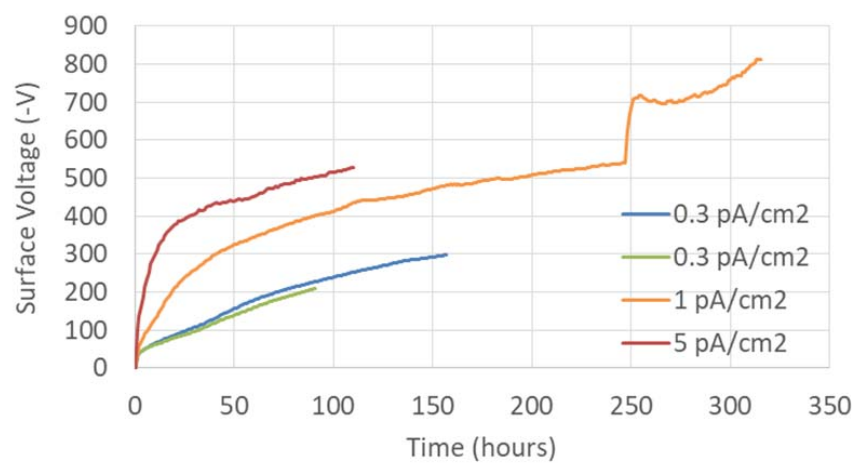

Fig. 4. Four exposures of Neoflon at three current levels. The medium current exposure was extended after $\sim 250 \mathrm{~h}$ with a high $(5 \mathrm{pA})$ current exposure.

levels $\left(0.3,1\right.$, and $\sim 0.15 \mathrm{pA} / \mathrm{cm}^{2}$, respectively). In all cases, the surface voltage reached the $3.4-\mathrm{kV}$ limit of the REEF Trek probe and the experiments were then halted. The results are shown in Fig. 3. The initial charging rates of the two unshielded exposures are approximately in proportion with the incident current levels, indicating that total conductivities are low and the charging response is close to linear. The shielded exposure does not fit this pattern, which we revisit in the simulations.

The charging response curves may appear linear; however, there is a slight curvature in each case, reflecting the combination of bulk and RIC in mitigating the build-up of electric field.

\section{B. Neoflon (FEP) Results}

Neoflon was initially irradiated at the "low" current level of $\sim 0.3 \mathrm{pA} / \mathrm{cm}^{2}$. The results revealed a nonlinear charging response that was unlike a classic exponential accumulation. This made the material a good candidate for investigating the possible effects of outgassing on the response. In a repeat experiment, a fresh identical Neoflon sample was allowed to sit in vacuum in the REEF chamber for 2 days prior to being exposed to the strontium-90 beta source. The response of this exposure was extremely similar to the previous sample, which had not allowed similar time for potential outgassing. We performed two further exposures of Neoflon, at the medium and higher current levels of 1 and $5 \mathrm{pA} / \mathrm{cm}^{2}$, respectively. The results are plotted (alongside the two other measurements above) in Fig. 4. 


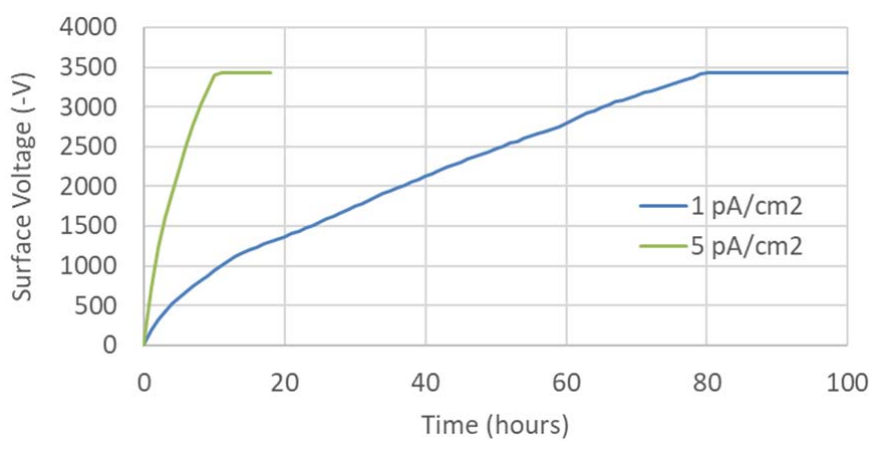

Fig. 5. Surface voltage measurements as a function of time for FR4 samples in REEF. The plateaus at $-3400 \mathrm{~V}$ are due to the limitation of the Trek probe.

It is clear that the charging response of this material is not a simple exponential approach to a plateau value of the surface voltage. Each curve is characterized by an initial rapid voltage gradient, followed by a more sedate (but still not necessarily exponential) charging profile. This is indicative of a complex structure of electron trapping depths and lifetimes in the dielectric. The $5 \mathrm{pA} / \mathrm{cm}^{2}$ extension is even more erratic as a rapid increase is followed by a dip in voltage, before charging appears to resume again. Unfortunately, we were not able to leave the experiment running for longer in order to try to observe a plateau. The implications of these complex behaviors are examined in this section on simulations.

\section{FR4 Results}

Two samples of the glass-reinforced epoxy material FR4 have been exposed, respectively, to 1 and $5 \mathrm{pA} / \mathrm{cm}^{2}$ charging currents in REEF. The charging response curves are shown in Fig. 5. In both cases, the maximum measurable voltage of $\sim 3.4 \mathrm{kV}$ was reached, and the experiments were discontinued. Both samples exhibited two phases of charging behavior in their response: an initial nonlinear phase with the obvious curvature, followed by a more linear phase that continued up to, and presumably beyond, the maximum observed surface voltage.

\section{PEEK Results}

The first PEEK sample was irradiated at the medium current of $1 \mathrm{pA} / \mathrm{cm}^{2}$. The response of this sample was different from all other materials in the campaign, as shown in Fig. 6. Having charged at a steady but nonlinear rate for a continuous period of $\sim 55 \mathrm{~h}$, the surface voltage of the sample reached a peak of $\sim 1300 \mathrm{~V}$ and then began to decline while still under irradiation at $1 \mathrm{pA} / \mathrm{cm}^{2}$. When this decline appeared to be plateauing at approximately half of the peak value, we extended the exposure by dramatically increasing the current level to the "high" value of $5 \mathrm{pA} / \mathrm{cm}^{2}$. As shown in the following, the surprising effect of this boost was a minimal impact on the surface voltage. Only a fractional increase of around $80 \mathrm{~V}$ was observed before the voltage peaked and began to decline again (ostensibly to a similar value as under $1 \mathrm{pA} / \mathrm{cm}^{2}$ irradiation). The observation that the surface voltage of a planar sample can peak and then decline while under continuous irradiation is not new [10]. However, the relatively small (and indeed transient) impact

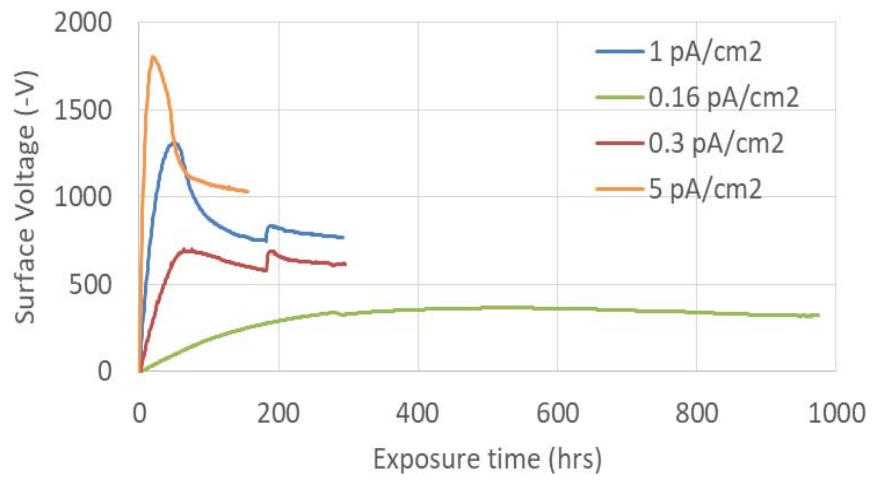

Fig. 6. Surface voltage measurements as a function of time for PEEK samples in REEF. The first sample (blue curve), which was initially irradiated at $1 \mathrm{pA} / \mathrm{cm}^{2}$, was irradiated under an increased current of $5 \mathrm{pA} / \mathrm{cm}^{2}$ after approximately $180 \mathrm{~h}$. One other sample (red curve), initially irradiated at $0.3 \mathrm{pA} / \mathrm{cm}^{2}$, was also extended with a $5 \mathrm{pA} / \mathrm{cm}^{2}$ exposure.

of the factor five increase in electron intensity represents a challenge for simulation codes. The second PEEK sample was irradiated at lower (shielded) current of $\sim 0.16 \mathrm{pA} / \mathrm{cm}^{2}$. A small interruption occurred after approximately $290 \mathrm{~h}$, due to a failure in the vacuum pump. However, the experiment was restarted with minimal disruption to the overall charging profile. As shown in Fig. 6, the surface voltage in this exposure peaked at approximately $360 \mathrm{~V}$, before declining slightly. A plateau of $\sim 320 \mathrm{~V}$ is implied by this decline, but as the irradiation had already continued for nearly $1000 \mathrm{~h}$, it was not possible to establish this more clearly.

The remaining two PEEK samples were irradiated at 0.3 and $5 \mathrm{pA} / \mathrm{cm}^{2}$, respectively. The former was extended with a $5 \mathrm{pA} / \mathrm{cm}^{2}$ exposure and experienced minimal additional charging like the first sample. Thus, we have observed consistency in the behavior of this material, both in terms of rising and falling voltage and also in terms of high current extensions. Indeed, the apparent "settled" voltages of the three unshielded current exposures are remarkably close together given the wide range in initial current levels. The shielded exposure stands apart and this is likely to be due to the altered energy profile, as well as absolute level, of the electron spectrum incident on the PEEK material sample.

\section{E. Relaxation Experiments}

In the absence of an incident electron current, the surface voltage of an irradiated dielectric may decrease rapidly. A simple model of this behavior would involve the voltage dropping exponentially, with a time constant determined by the bulk conductivity alone. In practice, the process is more complex, with various decay time constants superimposed on each other because of the presence of multiple electron trapping states in the dielectric. The influence of electric field strength on bulk conductivity also has an effect, as it does during charging [11]. We performed four relaxation experiments after the exposures of our PEEK samples. For software legacy reasons, these are not automated in REEF, and thus, the Trek probe measurements are far less frequent. The results from these experiments are presented in Fig. 7. Various time constraints meant that the duration varies from just $24 \mathrm{~h}$ to nearly $200 \mathrm{~h}$. The data from these relaxation curves are reasonably well fit by a single 


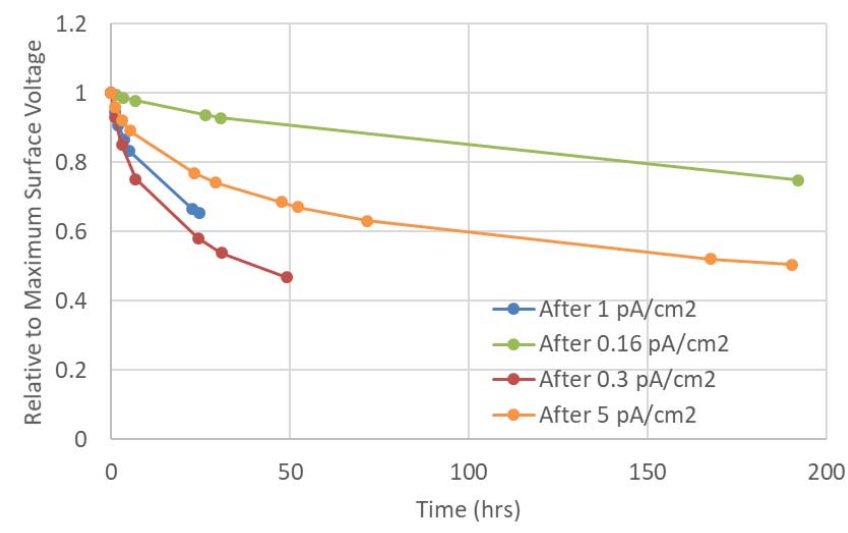

Fig. 7. Relaxation measurements with the four PEEK samples after irradiation at specified currents. Irregular surface voltage measurements are given as a fraction of the initial (maximum) voltage following exposure to Sr-90 electrons.

exponential with an offset asymptotic plateau. A possible physical interpretation of this is the superposition of (at least) one short-time-constant charge trapping state with (at least) one long-time-constant state. This is related to the so-called "delayed RIC" effect, where RIC decays only gradually once irradiation has ceased [8]. The wide range in relaxation time constants for the same material is not a simple function of irradiation current (and thus RIC), although it is notable that the slowest decay coincides with the longest and lowest current exposures $\left(\sim 1000 \mathrm{~h}\right.$ at $\left.\sim 0.16 \mathrm{pA} / \mathrm{cm}^{2}\right)$, which is consistent with a delayed RIC effect.

We also performed a single relaxation experiment each on Cirlex and Neoflon. In the former case, the retention of charge was extremely high, with only $\sim 2.5 \%$ decline in voltage after $120 \mathrm{~h}$ of relaxation. For Neoflon, by contrast, the surface voltage declined by $70 \%$ in $24 \mathrm{~h}$. As with PEEK, the decay profile was not a simple exponential with an asymptote at zero.

\section{Simulations}

We use three internal charging tools in order to compare our data with simulations. These are DICTAT (version 3) [12], MCICT [13], and NUMIT (version 2) [14]. The codes have different features, both in terms of how they are executed and the inputs and outputs associated with them. For DICTAT, we use the SPENVIS implementation [15], which is the most user-friendly of all the codes but has the restriction that the user input is limited to exposures of up to $100 \mathrm{~h}$ in duration. This limitation is inconsequential for the majority of REEF comparisons. MCICT implements the same underlying internal charging physics equations as DICTAT; however, MCICT uses the GEANT4 tool GRAS for electron transport and dose rate, which should be more accurate than the approximations contained within DICTAT. MCICT runs via command-line execution of a Python script, with parameters listed in an input file as described in [13]. The output information files are more comprehensive than DICTAT, and the key metric for comparison with REEF measurements - surface voltageremains a direct output. NUMIT 2.0 is maintained and distributed by NASA JPL and is operated via an independent executable file that requires separate input files for material parameters and spectral information. Unlike the other two codes, surface voltage is not a direct output and must be calculated by the user from the output information about the electric field strength broken down into ten sublayers of the dielectric material. NUMIT has been shown to be in good agreement with MCICT in terms of electron transport and dose rate [13] so it is expected that differences between the two codes' output are primarily due to the embedded physics equations used to calculate internal charging.

\section{A. Input Parameters}

The three simulation codes presented in this report share various input parameters associated with intrinsic physical and dielectric properties. Bulk conductivity (also called "dark" or "intrinsic" conductivity), RIC parameters $k_{\mathrm{p}}$ and $\Delta$ are listed for each material in Table I, as derived by experiments on identical samples at ONERA. The RIC parameters are provided by ONERA, using a stimulated charge relaxation technique (irradiation by high-energy penetrating electrons [16]). A description of the standard charging equations that use these parameters can be found in [5]. The molecular formula is a direct input for MCICT as it uses full Monte Carlo radiation transport to calculate electron penetration and dose rate. The formulas are known for three of the materials, though FR4 is estimated from an estimated composition of $60 \%$ fiberglass $\left(\mathrm{SiO}_{2}\right)$ and $40 \%$ epoxy. It is unlikely that small uncertainties in the elemental composition have a meaningful impact on simulated internal charging behavior. The values of effective $Z$ and $A$ are the required inputs for NUMIT and are derived using mass-weighted averages as recommended by the NUMIT user manual [9]. By contrast, in terms of physical properties, DICTAT requires only material density and sample thickness. NUMIT incorporates one additional parameterdelayed conductivity. As we have no empirical determination of this for our materials, and this is in any case generally associated with materials that have been previously exposed to radiation damage, we assume this parameter is 0 unless explicitly stated otherwise.

The incident spectrum is dealt with in slightly different ways by each code. MCICT can incorporate the stainless steel layer on the source pellet (and any additional shielding) directly, thereby accounting for the modification of the REEF spectrum shown in Fig. 2. NUMIT cannot have metal layers in its geometry (excepting grounding planes), so we used attenuated spectra calculated by MULASSIS as inputs to these simulations (i.e., the dashed curve shown in Fig. 2). DICTAT (as implemented in SPENVIS) is capable of including a single shielding layer in its input geometry. For the 11 unshielded exposures, we use a $0.075-\mathrm{mm}$ layer of material with density $8 \mathrm{~g} / \mathrm{cm}^{3}$ as the shield (reflecting the source packaging only), and for the two shielded exposures, we combine this with the $0.5-\mathrm{mm}$ aluminum shield to give a total equivalent shielding layer of $0.72 \mathrm{~mm}$ of the material with density $2.7 \mathrm{~g} / \mathrm{cm}^{3}$. These necessarily different approaches resulted in slightly different incident currents on the sample-0.21, 0.12 , and $0.16 \mathrm{pA} / \mathrm{cm}^{2}$ for DICTAT, NUMIT, and MCICT, respectively. This introduces a small additional level of uncertainty into our comparisons 


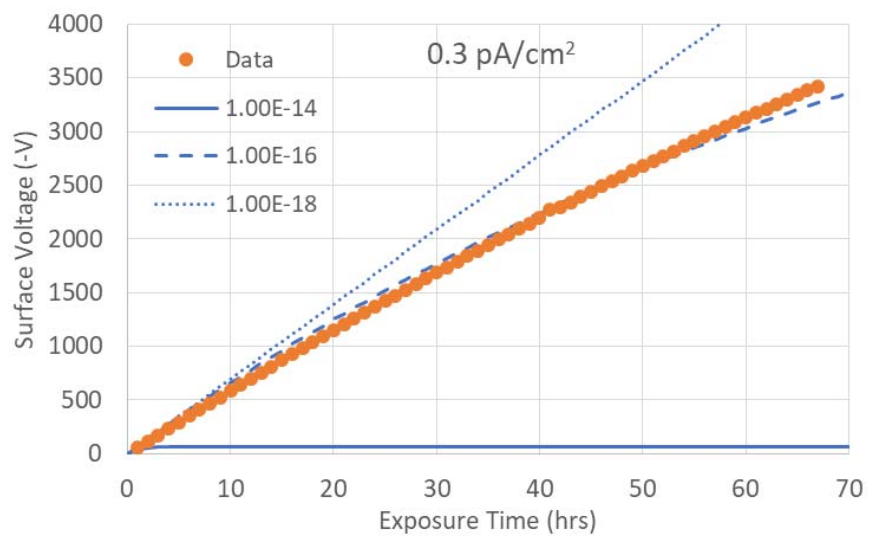

Fig. 8. MCICT simulations of Cirlex irradiated at $0.3 \mathrm{pA} / \mathrm{cm}^{2}$ with three different values for total conductivity in the range from $10^{-18}$ to $10^{-14} \mathrm{~S} / \mathrm{m}$. Closest agreement to data is found for an intermediate level of total conductivity of around $10^{-16} \mathrm{~S} / \mathrm{m}$.

between the codes, but one that would be encountered by anyone trying to use these codes to simulate charging behavior.

Activation energy is a required input for DICTAT and MCICT to account for temperature effects on conductivity (i.e., deviations from $298 \mathrm{~K}$ ). As our experiments are conducted at $310 \mathrm{~K}$ by default, this is a potentially important factor in the performance of simulations with respect to data. NUMIT does not have an allowance for such temperature variation, so this represents a potential flaw in any intercomparison between the codes. Discovering the correct activation energy for a material is also highly problematic. We have not been able to find published values for activation energy for any of the materials under test. Values between 1.2 and $1.5 \mathrm{eV}$ are, however, typical for polymers and other dielectrics [17]. According to the equations embedded within DICTAT and MCICT [15], at $310 \mathrm{~K}$, this would have the effect of increasing bulk conductivity by a factor between six and nine; however, other studies imply much weaker temperature dependence [8].

Although we do not have well-determined values of room temperature bulk conductivity for three of the four materials, previous experiments in REEF have shown that apart from extremely low currents (of the order of $0.01 \mathrm{pA} / \mathrm{cm}^{2}$ ), RIC is likely to dominate over bulk conductivity in terms of contribution to the total conductivity that shapes charging response. Therefore, we select a default value for bulk conductivity of $10^{-16} \mathrm{~S} / \mathrm{m}$ (for all materials except FR4) and use activation energy of zero for DICTAT and MCICT simulations. This is equivalent to using a lower value for room temperature bulk conductivity alongside a temperature of $310 \mathrm{~K}$ and a plausible value for activation energy. In either case, it is RIC that primarily determines total conductivity, so our default value for bulk conductivity can be thought of as an upper limit contribution to this total.

As an example of the effect of total conductivity on the simulations, Fig. 8 shows how the predictions from MCICT vary if bulk conductivity is set in the range from $10^{-18}$ to $10^{-14} \mathrm{~S} / \mathrm{m}$, with both the RIC and activation energy set to zero. At the lower end of this range, conductivity is so close to zero that the charging rate is approximately linear, and at the higher end, the conductivity is so high that the surface voltage plateaus at a very low level. It is implied by these data that the "true" value of total conductivity lies somewhere in between; however, this is a simplification.

\section{B. Simulation Results}

The results for each of the four materials are shown in the following in turn. To show the effect of RIC on the charging profiles, outputs are also shown for the case of $k_{\mathrm{p}}=0$. In all cases, the figure legends refer to the following combination of input parameters and codes:

1) empirical data (orange filled circles);

2) DICTAT, $\sigma_{i}=10^{-16} \mathrm{~S} / \mathrm{m} *$, and $k_{\mathrm{p}}=0$ (blue solid);

3) MCICT, $\sigma_{i}=10^{-16} \mathrm{~S} / \mathrm{m} *$, and $k_{\mathrm{p}}=0$ (blue dashed);

4) NUMIT, $\sigma_{i}=10^{-16} \mathrm{~S} / \mathrm{m} *$, and $k_{\mathrm{p}}=0$ (blue dotted);

5) DICTAT, $\sigma_{i}=10^{-16} \mathrm{~S} / \mathrm{m}$, and $k_{\mathrm{p}}=$ (see Table I) (red solid);

6) MCICT, $\sigma_{i}=10^{-16} \mathrm{~S} / \mathrm{m}$, and $k_{\mathrm{p}}=$ (see Table I) (red dashed);

7) NUMIT, $\sigma_{i}=10^{-16} \mathrm{~S} / \mathrm{m}$, and $k_{\mathrm{p}}=$ (see Table I) (red dotted); * except for FR4, where $\sigma_{i}=3 \times 10^{-15} \mathrm{~S} / \mathrm{m}$.

\section{Cirlex Simulations}

Simulations were performed for all the three runs plotted in Fig. 3 and these are plotted as shown in Fig. 9. The degree of agreement is mixed, with DICTAT performing poorest because, unlike MCICT and NUMIT, it does not agree well with the initial charging rate for the unshielded runs. This is likely due to the differences in electron transport, both through the stainless steel source lid and through the sample itself.

The inclusion of radiation-induced charging parameters introduces an additional curvature in the charging profiles, which is not reflected in the data. This implies that the RIC parameters derived by ONERA's experiments are too high. In the shielded exposure, at $\sim 0.16 \mathrm{pA} / \mathrm{cm}^{2}$, the agreement is poor for all codes, both in terms of initial charging rate and curvature (total conductivity). This is a significant problem as internal charging necessarily includes a calculation of electron attenuation in order to determine the incident current spectrum. Our simulations show that it does not seem to matter whether this calculation is performed independently of the codes or not as in all cases, the agreement with initial charging rate is poor.

\section{Neoflon Simulations}

Comparisons between data and simulations for Neoflon are plotted in Fig. 10. As the second run (at $0.3 \mathrm{pA} / \mathrm{cm}^{2}$ ) was a repeat of the first, we do not include those data separately. As the range of outputs is so wide, all Neoflon simulations are plotted on a logarithmic $y$-axis. We also note that NUMIT gave output errors for this material at the highest current level if RIC was included, presumably because the total conductivity exceeded an internal threshold. In any case, in all other simulations, NUMIT output was almost identical to MCICT output. In general terms, simulations exclusive of RIC overpredict the equilibrium surface voltage by an order of magnitude or more, and simulations inclusive of RIC underpredict the equilibrium surface voltage by a similar 

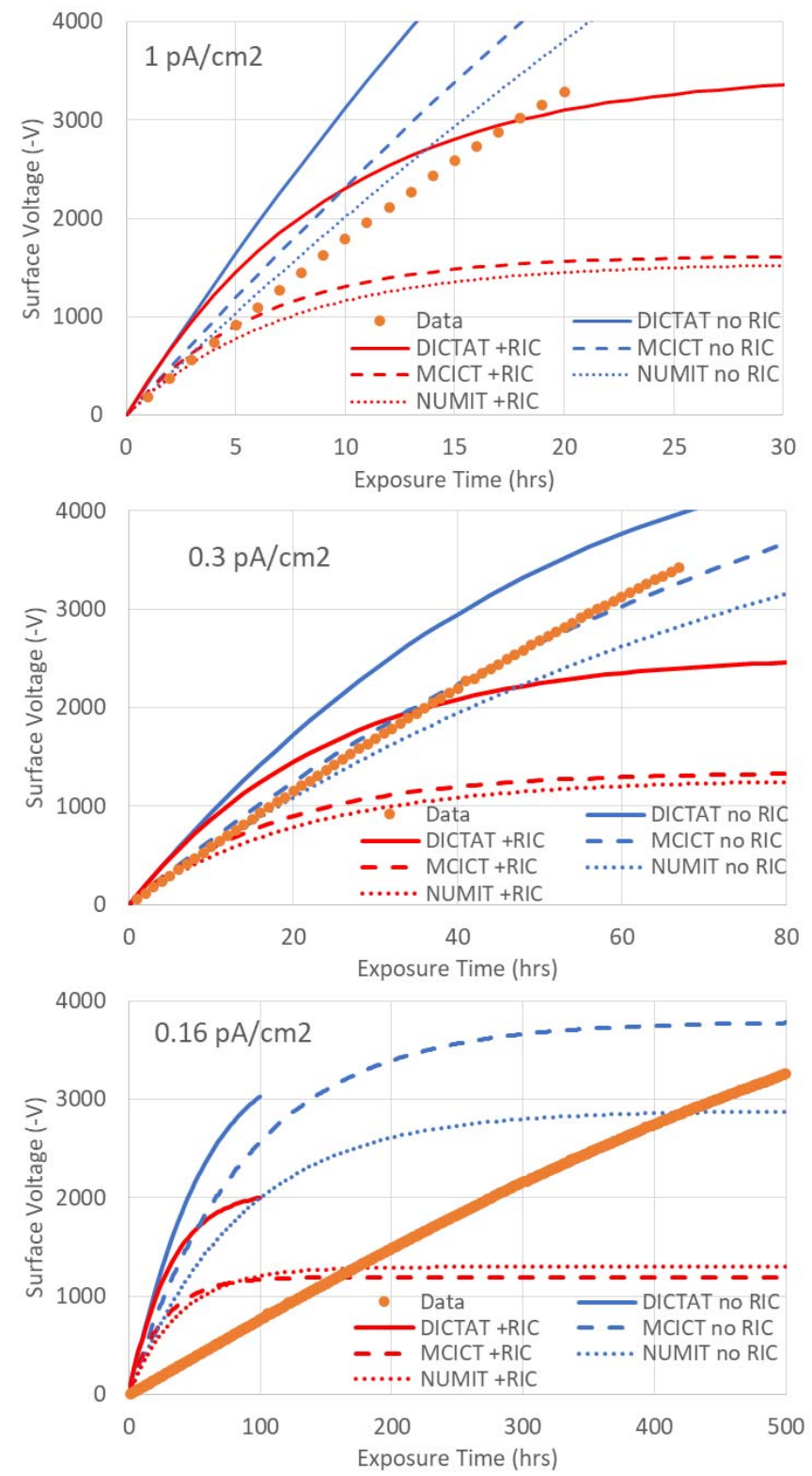

Fig. 9. DICTAT, MCICT, and NUMIT simulations of Cirlex surface voltage at $1.0 \mathrm{pA} / \mathrm{cm}^{2}$ (top), $0.3 \mathrm{pA} / \mathrm{cm}^{2}$ (middle), and $0.16 \mathrm{pA} / \mathrm{cm}^{2}$ (bottom) incident current. Simulations are shown inclusive (red lines) and exclusive (blue lines) of RIC. DICTAT runs on SPENVIS is time limited to $100 \mathrm{~h}$.

margin. This could be partially rectified by assuming a lower value for $k_{\mathrm{p}}$, leading to lower total conductivity. However, this would not resolve the issue of the shape, i.e., Neoflon does not follow a simple exponential profile with a single associated time constant (this is easier to see on the linear scale of Fig. 4). This is more likely to be due to multilevel charge trapping states that result in a superposition of time constants in the net charging profile. This is the charging equivalent of the nonideal exponential behavior of the relaxation experiments shown in Fig. 7, i.e., conductivity rises gradually after the onset of radiation, as it decays gradually with its removal.

The $5 \mathrm{pA} / \mathrm{cm}^{2}$ extension to the $1 \mathrm{pA} / \mathrm{cm}^{2}$ exposure resulted in an initial rapid rise in the surface voltage, followed by a small dip and then a more gradual rise. Unfortunately, we were
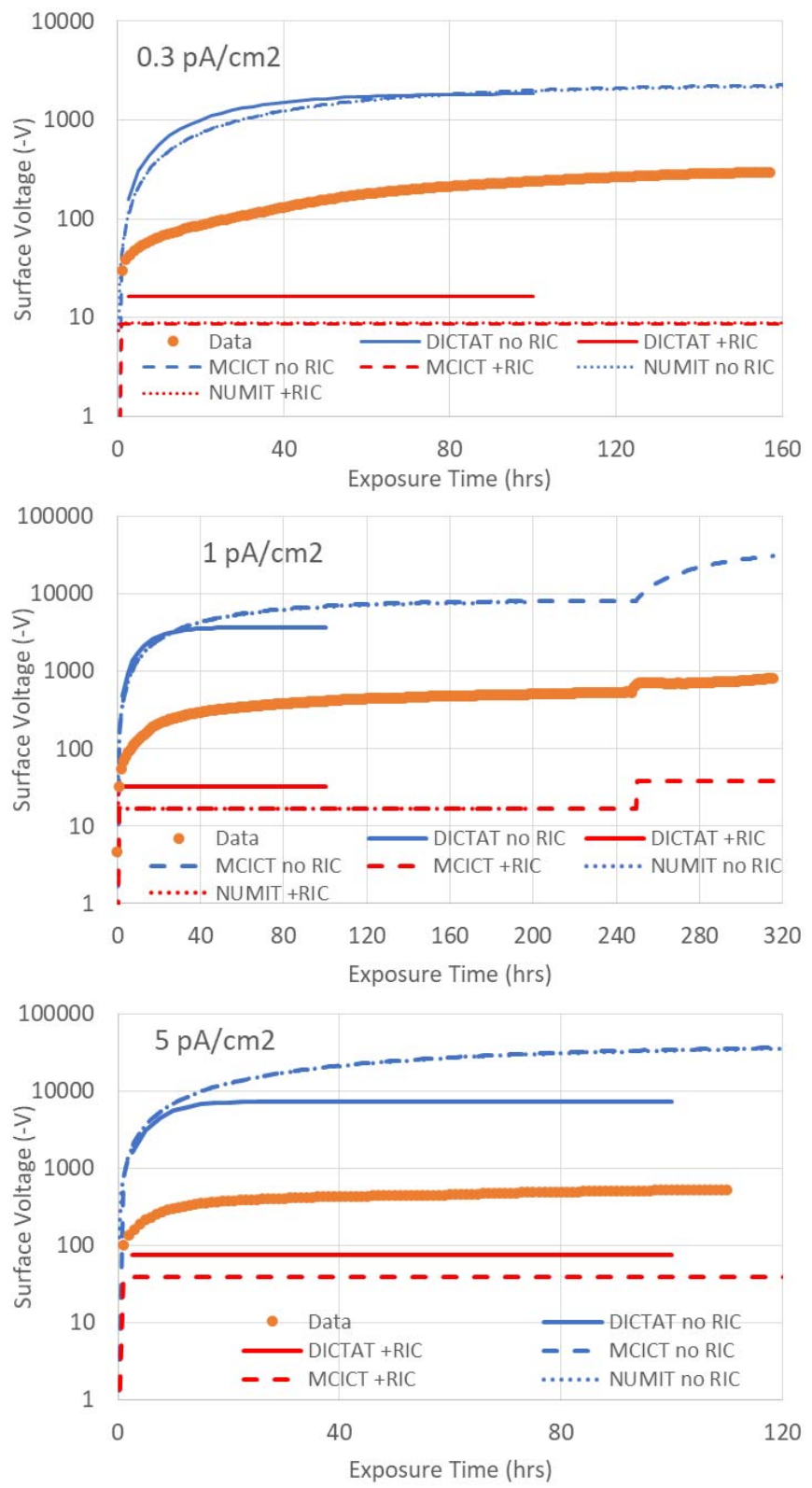

Fig. 10. DICTAT, MCICT, and NUMIT simulations of Neoflon surface voltage at $0.3 \mathrm{pA} / \mathrm{cm}^{2}$ (top), $1 \mathrm{pA} / \mathrm{cm}^{2}$ (middle), and $5 \mathrm{pA} / \mathrm{cm}^{2}$ (bottom) incident current. Simulations are shown inclusive (red lines) and exclusive (blue lines) of RIC. DICTAT runs on SPENVIS are time-limited to $100 \mathrm{~h}$. The $1 \mathrm{pA} / \mathrm{cm}^{2}$ run was extended with an increase to $5 \mathrm{pA} / \mathrm{cm}^{2}$ after $\sim 250 \mathrm{~h}$.

not able to leave this run going until equilibrium was reached. However, it is clear that this complex behavior is not recreated by the simulation tools, although the inclusion of RIC does reduce an otherwise very significant increase in surface voltage that it is not seen in the data.

\section{E. FR4 Simulations}

Comparisons between data and simulations for the two FR4 runs are plotted in Fig. 11. Unlike other materials, RIC is not included and we use the value derived by ONERA for bulk conductivity $-3 \times 10^{-15} \mathrm{~S} / \mathrm{m}$. Although the data are limited, we do not find good agreement with simulations from any of the codes, which do at least agree relatively well with each other. Rather like Neoflon, the charging profile of 

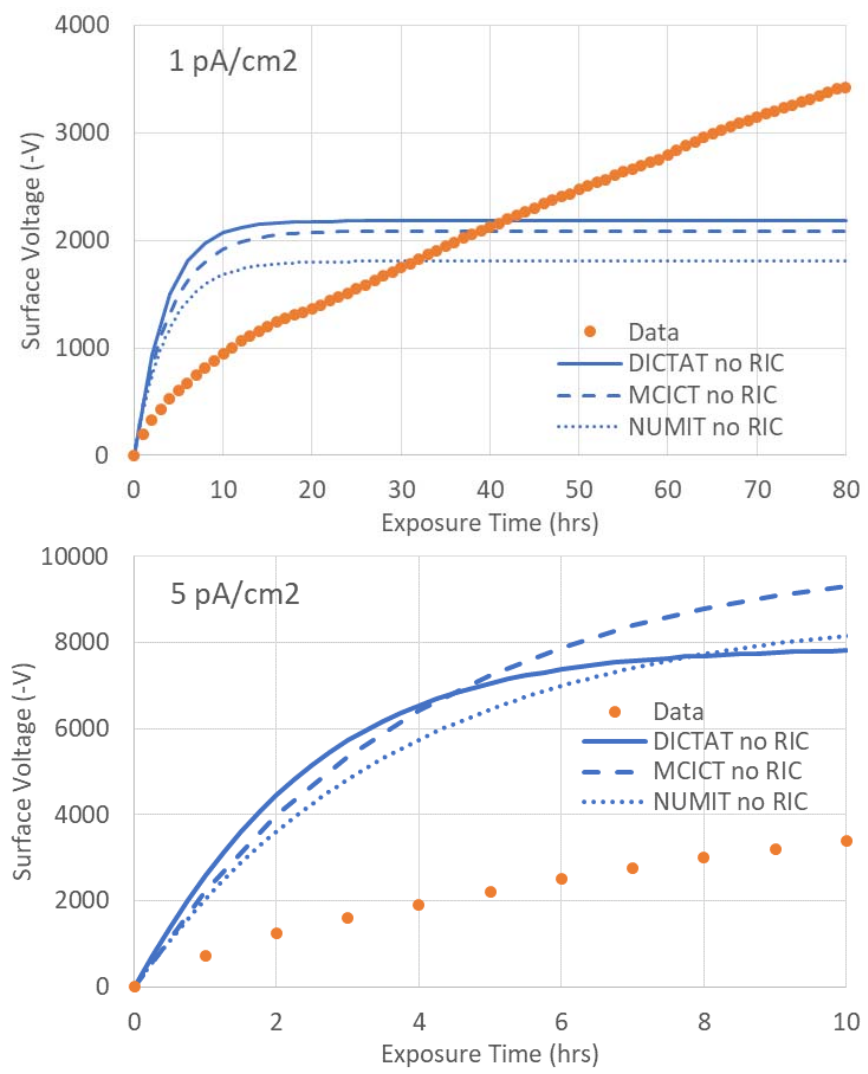

Fig. 11. DICTAT, MCICT, and NUMIT simulations of FR4 surface voltage at $1 \mathrm{pA} / \mathrm{cm} 2$ (top) and $5 \mathrm{pA} / \mathrm{cm} 2$ (bottom) incident current. Simulations are shown exclusive of RIC as this is zero for FR4.

FR4 (at least at $1 \mathrm{pA} / \mathrm{cm}^{2}$ ) is rapid at first but then appears to become almost linear. This cannot be a dose effect (see the delayed conductivity discussion later on) as it occurs early on irradiation. Therefore, we believe it is more likely to be because of a multispeed charging effect due to multilevel traps with associated constants.

The value for room temperature bulk conductivity of $3 \times$ $10^{-15} \mathrm{~S} / \mathrm{m}$ appears to lead to a greater degree of curvature than is observed in the data, implying that this value is too high. Given that measurements were taken at $310 \mathrm{~K}$, at which temperature the bulk conductivity would be expected to be even higher, this divergence between the prediction and measurement could be even greater than plotted in Fig. 11.

\section{F. PEEK Simulations}

Comparisons between data and simulations for PEEK are plotted in Fig. 12. The PEEK results are unique, in which the duration of the experiments was sufficient to show reductions in surface voltage prior to equilibrium being reached (we, of course, cannot tell if this would have been the case with other samples where equilibrium was not reached). This effect cannot be replicated by DICTAT or MCICT, whose output steadily approaches a maximum at equilibrium. However, NUMIT has the capability of including delayed conductivity, i.e., additional conductivity as a function of cumulative dose rather than instantaneous dose rate [18]. We discuss this in Section IV-G. In general terms, both the MCICT and NUMIT produce a good agreement with observed PEEK charging
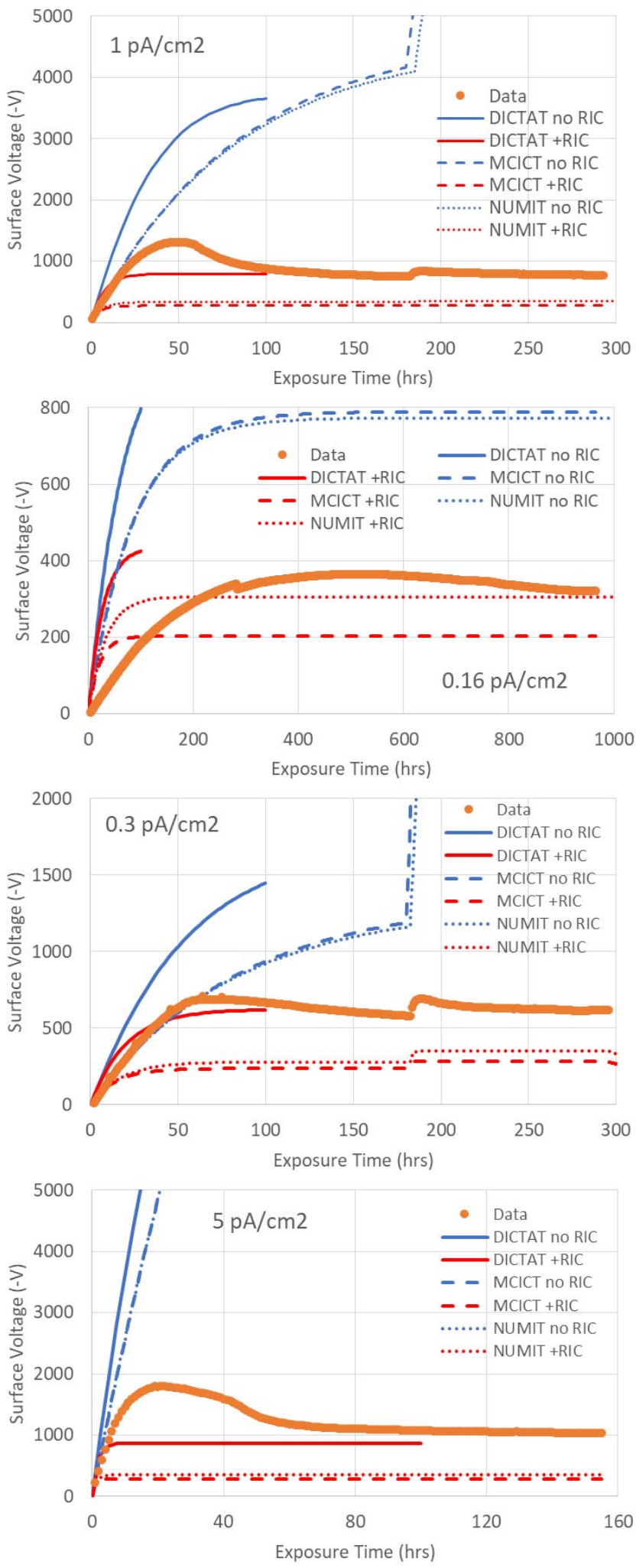

Fig. 12. DICTAT, MCICT, and NUMIT simulations of PEEK surface voltage at $1 \mathrm{pA} / \mathrm{cm}^{2}$ (top), $0.16 \mathrm{pA} / \mathrm{cm}^{2}$ (second), $0.3 \mathrm{pA} / \mathrm{cm}^{2}$ (third), and $5 \mathrm{pA} / \mathrm{cm}^{2}$ (bottom) incident current. Simulations are shown inclusive (red lines) and exclusive (blue lines) of RIC. DICTAT runs on SPENVIS is time limited to $100 \mathrm{~h}$. The 0.3 and $1 \mathrm{pA} / \mathrm{cm}^{2}$ runs were extended with an increase to $5 \mathrm{pA} / \mathrm{cm}^{2}$ after $\sim 180 \mathrm{~h}$.

profiles in the initial phases (the exception again being the shielded exposure at $\left.0.16 \mathrm{pA} / \mathrm{cm}^{2}\right)$. Simulations excluding RIC maintain this agreement longer than those including RIC. 

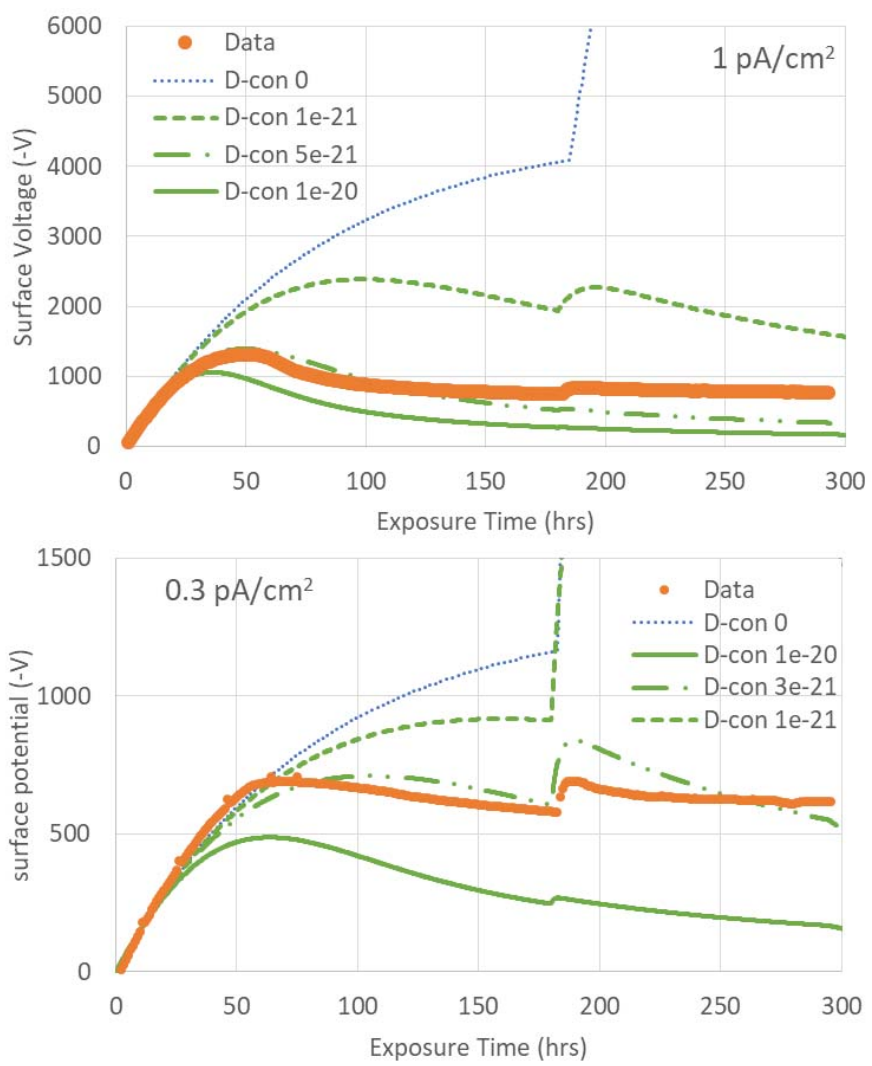

Fig. 13. NUMIT simulations of PEEK surface voltage at $1 \mathrm{pA} / \mathrm{cm}^{2}$ (top) and $0.3 \mathrm{pA} / \mathrm{cm}^{2}$ (bottom), and $5 \mathrm{pA} / \mathrm{cm}^{2}$ incident current. RIC parameters are not included, but delayed conductivity is included at three alternative levels in the range from $10^{-21}$ to $10^{-20} \mathrm{~S} / \mathrm{m} / \mathrm{rad}$.

However, the equilibrium surface voltage is better represented by the simulations that include RIC. Therefore, it is difficult to judge the validity of the RIC parameters deduced by experiments at ONERA, although the behavior clearly cannot be explained by bulk conductivity alone.

\section{G. Delayed Conductivity}

We repeated our NUMIT simulations for PEEK with the option of delayed conductivity included in the input file. Note that this is a process where conductivity increases with dose (also known as "aging") during irradiation and is thus distinct from delayed RIC, which applies after irradiation has ceased [19]. Having no prior knowledge of what this parameter might be for this material, we used a range of values between $10^{-21}$ and $10^{-20} \mathrm{~S} / \mathrm{m} / \mathrm{rad}$ in our simulations, which excluded RIC as this appears to lead to an underestimate in equilibrium surface voltage even in the absence of delayed conductivity. The results are plotted in Fig. 13 for the examples of 1 and $0.3 \mathrm{pA} / \mathrm{cm}^{2}$ (for which the improvement due to the inclusion of delayed conductivity is the greatest).

The value of delayed conductivity that produces the best agreement with data (albeit in the absence of RIC) appears to be in the range of $\sim 3-5 \times 10^{-21} \mathrm{~S} / \mathrm{m} / \mathrm{rad}$. Although these comparisons cannot conclusively establish accurate parameters for delayed conductivity, they do imply that for PEEK, this component of the charging dynamics is a very important capability for a charging simulation tool to have. The uniqueness of

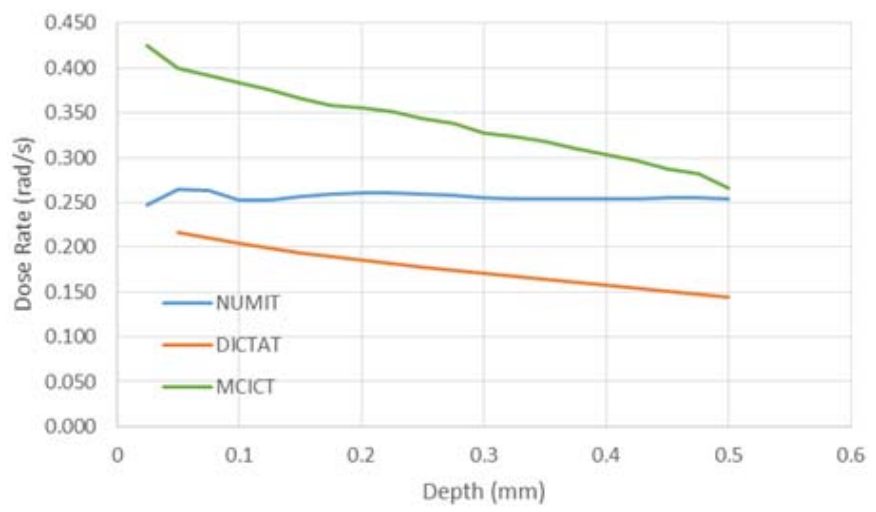

Fig. 14. Examples of dose rate profile in a sample of PEEK. Dose rates are shown as a function of depth for an incident REEF current of $1 \mathrm{pA} / \mathrm{cm}^{2}$ for each of the three simulation codes.

NUMIT in the three tools we have tested is, therefore, notable.

\section{Discussion}

The general conclusion of this paper must be that it is extremely hard to find agreement between empirical data and simulations over these long charging timescales. We identify four key explanations for the mismatches we find between prediction and observation.

1) Electron Transport: The incident spectrum is crucial to determining the initial charging rate, which, in conjunction with total conductivity, also helps determine the equilibrium surface voltage.

2) RIC Parameters: These are often unknown or poorly constrained for dielectric materials, which limits the predictive ability of simulations. However, even with parameters extracted from bespoke experiments on identical samples, we struggle to replicate observed charging behavior.

3) Multilevel Trapping: The simplistic model of a single time constant for charging or relaxation (apropos of delayed RIC) underestimates the complexity of charge trapping in dielectrics.

4) Delayed Conductivity: This additional factor may be one of many second-order effects that affect charging behavior (light-stimulated electron emission is another, although this is less important for thicker samples). Our PEEK example shows how the inclusion of this example can significantly improve the prediction of charging behavior.

The first item in this list is perhaps the easiest to quantify. For the outputs of the respective codes, it is possible to compare the passage of electron radiation through the dielectric samples. For example, Fig. 14 shows the dose rates as a function of depth in PEEK samples, according to the three codes under test. There are clearly significant differences both in terms of the shape and normalization, which affects the charging dynamics through the link to RIC where $\sigma_{\text {RIC }}=$ $k_{\mathrm{p}} \cdot \dot{D}^{\Delta}$ (where $\dot{D}$ is the dose rate).

Even in the absence of RIC, accurately calculating electron transport is important as it affects the current distribution within the dielectric sample and thus the initial charging rate. 
Taken together, the radiation transport aspects of internal charging tools are an important factor as the dielectric properties of the materials themselves.

The balance between the bulk conductivity, temperature effects, and RIC cannot be fully disentangled with these data. Material parameters provided by ONERA for these samples, including for RIC, show mixed results in terms of improving agreement with measurements, and without the key parameter of bulk conductivity, it is very difficult to judge whether these parameters are correct. It is not within the scope of this paper to establish an alternative number for dielectric properties of the materials that lead to better agreement with simulations. Such a task would require many more experimental exposures with various carefully controlled permutations of ambient conditions (flux and temperature). Fundamentally, it is important to note that even in constant current irradiations, conductivity is not likely to be constant.

\section{CONCLUSION}

We have shown that the long-duration low-intensity measurements in REEF cannot simply be recreated with simulation codes in the absence of a number of well-constrained parameters. Even with full parametric flexibility, it is only possible to fit the simplest of charging profiles, i.e., those that are consistent with a single exponential time constant. With sufficient data, it may be possible to regressively fit a multiparameter model that includes RIC, delayed conductivity, and the other aspects mentioned above. However, none of these codes are capable of doing this deconvolution rather they are set up for the forward calculation of electric fields given known parameters. This emphasizes the need for a common database of material dielectric properties that can be used to find standard inputs to simulation tools. In addition, ongoing efforts to develop new codes based on solid-state physics should be encouraged, as these may help to improve the capacity of models to replicate complex charging behavior [20].

The complex range of results from our experiments may suggest that an experimental approach has higher fidelity than a modeling approach, especially where specific materials are of interest to be used in an electron environment. Certainly, we can say that simulations alone, without empirical validation, should be not treated as automatically reliable indicators of the true internal charging hazard.

\section{REFERENCES}

[1] G. L. Wrenn, D. J. Rodgers, and K. A. Ryden, "A solar cycle of spacecraft anomalies due to internal charging," Ann. Geophys., vol. 20, pp. 953-956, Jul. 2002.
[2] P. G. Coakley, M. J. Treadaway, and P. A. Robinson, "Low flux laboratory test of the internal discharge monitor (IDM) experiment intended for CRRES," IEEE Trans. Nucl. Sci., vol. NS-32, no. 6, pp. 4065-4072, Dec. 1985.

[3] A. R. Frederickson and J. R. Dennison, "Measurement of conductivity and charge storage in insulators related to spacecraft charging," IEEE Trans. Nucl. Sci., vol. 50, no. 6, pp. 2284-2291, Dec. 2003.

[4] K. A. Ryden, P. A. Morris, D. J. Rodgers, R. Bielby, P. R. Knight, and J. Sorensen, "Improved demonstration of internal charging hazards using the realistic electron environment facility (REEF)," in Proc. IEEE 8th Spacecraft Charging Technol. Conf., Huntsville, AL, USA, Oct. 2003. pp. 20-24

[5] A. Hands and K. Ryden, "Experimental measurement of low-intensity and long-duration internal charging behavior," IEEE Trans. Plasma Sci., vol. 45, no. 8, pp. 1938-1946, Aug. 2017.

[6] F. Lei et al., "MULASSIS: A geant4-based multilayered shielding simulation tool," IEEE Trans. Nucl. Sci., vol. 49, no. 6, pp. 2788-2793, Dec. 2002.

[7] W. Guo, M. Dufault, S. B. Cahn, J. A. Nikkel, Y. Shin, and D. N. McKinsey, "Scintillation and charge yield from the tracks of energetic electrons in superfluid helium-4," J. Instrum., vol. 7, no. 1, p. 01002, 2012.

[8] T. Paulmier, B. Dirassen, M. Belhaj, and D. Rodgers, "Charging properties of space used dielectric materials," IEEE Trans. Plasm. Sci., vol. 43 , no. 9, pp. 2894-2900, Sep. 2015.

[9] K. Wousik, "NUMIT 2.0 user manual," NASA JPL, La Cañada Flintridge, CA, USA, Tech. Rep., 2014.

[10] P. Morris, "Materials charging effects under extreme environments (MATCHE3)," QinetiQ, Farnborough, U.K., Final Rep. QINETIQ/TS/AS/TN1300174, 2013.

[11] J. Adamec and J. H. Calderwood, "Electrical conduction in dielectrics at high fields," J. Phys. D: Appl. Phys, vol. 8, no. 5, pp. 551-560, 1975.

[12] D. J. Rodgers, K. A. Ryden, P. M. Latham, G. L. Wrenn, L. Levy, and B. Dirassen, "Engineering tools for internal charging, final report on ESA contract," Defence Eval. Res. Agency, Farnborough, U.K., Tech. Rep. 12115/96, 2000.

[13] F. Lei, D. Rodgers, and P. Truscott, "MCICTâE"Monte-Carlo internal charging tool," in Proc. IEEE 14th Spacecraft Charging Technol. Conf., Noorwijk, 2016

[14] I. Jun, H. B. Garrett, W. Kim, and J. I. Minow, "Review of an internal charging code, NUMIT," IEEE Trans. Plasma Sci., vol. 36, no. 5, pp. 2467-2472, Oct. 2008 .

[15] Spenvis Help Page. Accessed: Jun. 22, 2018. [Online]. Available: www.spenvis.oma.be/help/background/charging/dictat/dictatman.html

[16] T. Paulmier et al., "Aging effect and induced electric phenomena on dielectric materials irradiated with high energy electrons," IEEE Trans. Plasma Sci., vol. 41, no. 12, pp. 3422-3428, Dec. 2013.

[17] Publications and Presentations Database. Accessed: May 30, 2018. [Online]. Available: http://polymerdatabase.com/polymer\%20chemistry/ Ea.html

[18] Space Engineering, Spacecraft Standard, Standard ECSS-E-50-14A, Dec. 2008.

[19] L. Levy, T. Paulmier, B. Dirassen, C. Inguimbert, and M. Van Eesbeek, "Aging and prompt effects on space material properties," IEEE Trans. Plasma Sci., vol. 36, no. 5, pp. 2228-2237, Oct. 2008.

[20] R. Pacaud, T. Paulmier, P. Sarrailh, D. Payan, K. Ryden, and A. Hands, "Study of internal charging of polymers through numerical and experimental analyses - Comparison of different space numerical tools," $J$. Appl. Phys., vol. 125, 2019, Art. no. 045108. doi: 10.1063/1.5055221.

Authors' photographs and biographies not available at the time of publication. 